\title{
Efectividad de los jugadores de futsal según sus posiciones Effectiveness of futsal players according to their positions
}

\author{
Javier Alvarez Medina, Javier Ramírez San Jose, Victor Murillo Lorente
}

Universidad de Zaragoza (España)

\begin{abstract}
Resumen. Introducción: La lógica interna del futbol sala está formada por ataque y defensa que implican continuas transiciones ofensivas y defensivas y ocasiones de gol. Esta gran movilidad en ataque tiene como finalidad provocar y aprovechar un desequilibrio en el esquema táctico defensivo contrario, creando así un efecto sorpresa de incertidumbre que genere una opción clara de finalización y así poder anotar gol. Existen estudios que explican cómo, cuándo se consiguen los goles pero no existe ninguno que diga quién los consigue. El objetivo de este trabajo es saber cómo se distribuyen los goles entre los diferentes tipos de jugadores en las mejores ligas europeas de fútbol sala. Método: Estudio cuantitativo, descriptivo. La muestra asciende a 110 partidos en la liga italiana (11 equipos), 240 en la liga española (16 equipos) y 273 en la liga rusa (14 equipos), lo que supone un total de 623 partidos de los que se han podido analizar 619 y una muestra (n) alcanzada de 4716 goles de la temporada 2.014-2.015. Para la recogida de datos se han utilizado las actas de los partidos. Datos descriptivos dados en frecuencias, medias y porcentajes y estadística inferencial utilizando Chi-cuadrado para establecer la relación entre variables. Resultados y conclusiones: Los resultados establecen que existe una relación directa entre las posiciones de partida y la consecución de goles. Así, el pívot obtiene un 40,37\% de los goles, seguido por los alas con un 37,24\%, los cierres con un 19,64\%.

Palabras clave: Futsal, goles, posiciones, pívot, ala, cierre, portero.
\end{abstract}

Abstract. Introduction: The internal logic of futsal consists of attack and defense involving continuous offensive and defensive transitions and goal chances. This great mobility in attack aims to provoke and take advantage of an imbalance in the opposing defensive tactical scheme, creating a surprise uncertainty effect that generates clear chances of finishing and thus scoring goals. There exist studies explaining how, or when, goals are achieved; however, none discusses who gets them. The objective of this paper is to assess how goals are distributed among the different types of players in the best European futsal leagues. Method: Quantitative, descriptive study. The sample consists of 110 matches in the Italian league (11 teams), 240 in the Spanish league (16 teams), and 273 in the Russian league (14 teams), with a total of 619 matches analyzed on a total of 623 available, and a sample (n) of 4,716 goals scored during the season 20142015. For the collection of data, match minutes were used. Descriptive data was presented in frequencies, means and percentages, whilst inferential statistics were run using Chi-square to establish the relationship between variables. Results and conclusions: The results establish that there is a direct relationship between starting positions and achievement of goals. Pivots get $40.37 \%$ of the total goals, followed by wings with $37.24 \%$, and closures with $19.64 \%$.

Key words: Futsal, goals, positions, pivot, wing, closing, goalkeeper.

\section{Introducción}

La lógica interna del futbol sala está formada por ataque y defensa que implican continuas transiciones ofensivas y defensivas y ocasiones de gol (Pascual, Álvarez, Carbonel, \& Pérez, 2019; Schneider, Da Cunha Voser, \& Eloi, 2015). Las dimensiones del terreno de juego (40x20m), así como la no existencia de zonas prohibidas, condiciona el juego permitiendo una gran movilidad que hace que los jugadores puedan acabar en posiciones distintas en cada jugada. Esta gran movilidad en ataque tiene como finalidad provocar y aprovechar un desequilibrio en el esquema táctico defensivo contrario, creando así un efecto sorpresa de incertidumbre que genere una opción clara de finalización y así poder anotar gol.

Cada vez hay más estudios en otros deportes colectivos que intentan explicar cómo y cuándo se consiguen los goles (Argudo, García, \& Ruiz, 2016; Jiménez-Olmedo, EspinaAgullo, \& Manchado, 2017; Martínez, \& González, 2019), un objeto de estudio también muy estudiado en los últimos año en fútbol sala (Álvarez, Murillo, \& García, 2018; Álvarez, Murillo, García, \& Parra, 2018; Campos, Pellegrinotti, Pasquarelli, Rabelo, Santa Cruz, \& Gómez, 2015; Grela, 2013; Lapresa, Álvarez, Arana, Garzón, \& Caballero, 2013; Pascual,

Fecha recepción: 25-09-18. Fecha de aceptación: 18-07-19

Victor Murillo Lorente

victormurillo1301@hotmail.com
Álvarez, Carbonel, \& Pérez, 2019; Sampedro, \& Prieto 2011). Así, la media de goles en la liga española profesional de fútbol sala está entre 3,22 y 4 por equipo/partido, obteniendo en la temporada 2.013-2.014 un total de 1.355 goles, con una media por equipo de $90,40 \pm 27,72$ goles y por partido de 3,22(Álvarez, Murillo, \& García, 2018; Álvarez, Murillo, García, \& Parra, 2018). No se ha encontrado ningún estudio que diga quién, qué tipo de jugador mete los goles. Caso especial es la figura del denominado portero-jugador, que aparece con las modificaciones hechas en el año 2.006 en el reglamento, y que busca tener superioridad numérica que permita provocar con más facilidad ocasiones de gol o tener una mayor posesión del balón. Esta situación táctica ha sido objeto de estudio en las mejores ligas del mundo, la española (Álvarez, Ramírez, \& Murillo, 2019) y brasileña (Da Cuna Voser, Goncalves, \& Eloi, 2016) si bien responde a una táctica utilizada para saber cuántos goles se consiguen con ella pero no para saber quién los consigue.

Los jugadores de las plantillas de los clubes están clasificados, conforme a las directrices de la liga estatal donde participan, según su posición inicial en el campo y sus características de juego diferenciando entre portero, cierre, ala y pívot. El éxito de un equipo comienza con la confección de una plantilla equilibrada(Álvarez, Manonelles, Giménez, \& Nuviala, 2009), por lo que será importante saber qué grado de responsabilidad en la consecución de los goles debe tener cada posición y tipo de los jugadores de la plantilla.

Además, teniendo presente que el fútbol sala es un deporte moderno, en el cual España ocupa el número 1 en el 
ranking de la Unión de Asociaciones Europeas de Fútbol (UEFA) obteniendo 7 de los 10 Campeonatos de Europa que ha organizado desde 1.996 a 2.016(RFEF, 2009), hace de este país exportador, tanto de jugadores como de técnicos por Europa y el resto del mundo, por lo que al plantearse el objeto de estudio surge la pregunta ¿existen diferencias entre las principales ligas profesionales de fútbol sala Europeas?

Los objetivos de este trabajo son:

- Conocer cómo se distribuyen los goles entre los diferentes tipos de jugadores que forman un equipo.

- Establecer si existen diferencias entre las mejores ligas europeas.

\section{Material y método}

Estudio retrospectivo cuantitativo, descriptivo y comparativo de quién mete los goles en las tres mejores ligas profesionales europeas de fútbol sala: española, italiana y rusa.

La muestra asciende a la totalidad de la población objeto de estudio, que corresponde a 110 partidos en la liga italiana con un total de 11 equipos, 240 en la liga española con 16 equipos y 273 en la liga rusa con 14 equipos, lo que supone un total de 623 partidos de los que se han podido analizar 619 y una muestra ( $n$ ) alcanzada de 4716 goles.

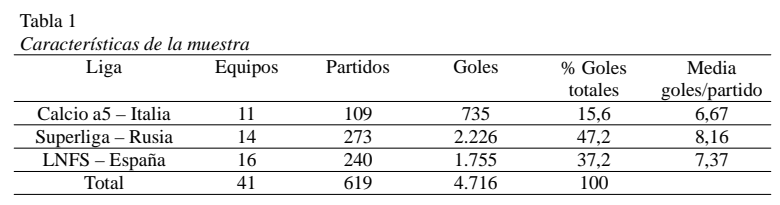

\section{Justificación de la muestra}

Se han elegido las tres mejores ligas europeas, según títulos europeos, por considerar que son la mejor referencia para estudiar el objeto de estudio. La Unión de Asociaciones Europeas de Fútbol (UEFA) ha organizado 10 Campeonatos de Europa desde 1.996 a 2.016, siendo los países que más títulos han obtenido España con 7, Italia con 2 y Rusia con 1.

\section{Clasificación de los jugadores según su posición ini- cial en el campo}

Además del portero, según la posición inicial que se ocupa en el campo se distinguen 3 posiciones (Álvarez, 2015):

- Cierre: jugador más próximo a la portería. En él prevalecen las características defensivas, como organizar la colocación de los compañeros, coberturas, ayudas y anticipación.

- Ala derecha e izquierda: ocupa una posición más avanzada, poseyendo características más ofensivas que el cierre como tiro y regate más rápido, además de la capacidad de desmarque y asistencia a los compañeros. Actúa, generalmente, por la derecha o por la izquierda.

- Pívot: es el jugador más próximo a la portería adversaria en el que predominan características ofensivas, como un buen tiro, regate, manejo, capacidad de asistir a compañeros y de realizar desmarques.

La liga italiana es la única que añade a la clasificación anterior la categoría de «universal» definido como aquel que puede jugar en cualquiera de las tres posiciones de campo ya definidas.

Atendiendo a la alta movilidad de los jugadores en este deporte en los partidos se entremezclan cualidades de una y otra posición dando lugar a: cierre-ala, ala-cierre, ala-pívot. Caso aparte es la situación de juego denominada «porterojugador o jugador-portero», que busca tener una superioridad numérica en ataque, donde el que ocupa la posición de portero en ataque actúa como un jugador de campo más. Esta situación no se va a tener en cuenta en el por no estar establecida por las ligas como clasificación de los jugadores.

\section{Recogida de datos}

La recogida de datos se hizo a través de las Actas finales de los partidos. Se pidió permiso a la liga rusa, española e italiana para el acceso a las mismas, el cual fue concedido y remitidos a la web oficial donde están publicadas las mismas, siendo el acceso público a los datos registrados como: quién mete los goles, minuto del gol, tarjetas amarillas, tarjetas rojas y otros como jugadores titulares, convocados, entrenadores, árbitros y sede donde se disputa el partido.

Para la clasificación de los jugadores se consultó la página web oficial de cada liga que remitió a las respectivas webs de cada club donde salen las plantillas clasificadas por puestos.

\section{Instrumentos de análisis de datos}

Paquete estadístico SPSS, versión 22.0, para la estadística descriptiva e inferencial en la búsqueda de relaciones asociativas entre variables categóricas. Datos descriptivos dados en frecuencias, medias y porcentajes y estadística inferencial a través de tablas de contingencia utilizando las pruebas no paramétricas Chi-cuadrado para establecer la relación entre variables estableciendo significación estadística cuando el valor es Â0,05.

\section{Resultados}

El cruce de variables se ha realizado solo entre la liga española y rusa por existir en la liga italiana la variable extra jugador universal.

Clasificación de los jugadores según su posición inicial en el campo-confección plantilla

Los jugadores han quedado clasificados como portero un $13,69 \%$, ala un $36,4 \%$, cierre $20,7 \%$, pívot $26,71 \%$ y universal un 2,5\% (solo se da en la liga italiana) (tabla 2).

Según la liga estudiada:

- Liga Española: 15,12\% portero, 47,89\% ala, 19,32\% cierre, $17,64 \%$ pívot.

- Liga Rusa: $11,76 \%$ portero, 19,1\% ala, 25\% cierre, 44,1\% pívot.

- Liga Italiana: $14,01 \%$ portero, $41,4 \%$ ala, $17,2 \%$ cierre, 17,83\% pívot y 11,11\% universal.

Con estos resultados dados en porcentaje una plantilla tipo de 14-15 jugadores quedaría formada por 2 porteros, 5 alas, 3 cierres, 4 pívots y 0 universales (tabla 3).

En las diferentes ligas:

- $\quad$ Española: 2 porteros, 7 alas, 3 cierres y 3 pívots.

- Rusa: 2 porteros, 3 alas, 4 cierres y 6 pívots.

- Italiana: 2 porteros, 6 alas, 2 cierres, 3 pívots y 2 universales. 


\section{Goles por posiciones}

Un $0,63 \%$ de los goles los marcan los porteros, un 37,24\% los alas, un 19,64\% los cierres, un 40,37 los pívots y un 2,12 los universales (solo tenidos en cuenta en la liga italiana) (tabla 4).

Por ligas se ha obtenido:

- Liga Española: 0,5\% portero, 54,1\% ala, 15,8\% cierre, 29,5\% pívot.

- $\quad$ Liga Rusa: 0,8\% portero, 21,9\% ala, 24,7\% cierre, 52,6\% pívot.

- Liga Italiana: 0,4\% portero, 43,3\% ala, 13,4\% cierre, $29,1 \%$ pívot y $13,7 \%$ universal.

Se obtienen diferencias significativas para todos los jugadores de campo de la misma liga; Ala-Cierre $(0,000)$, AlaPívot $(0,000)$ y Pívot-Cierre $(0,047)$.

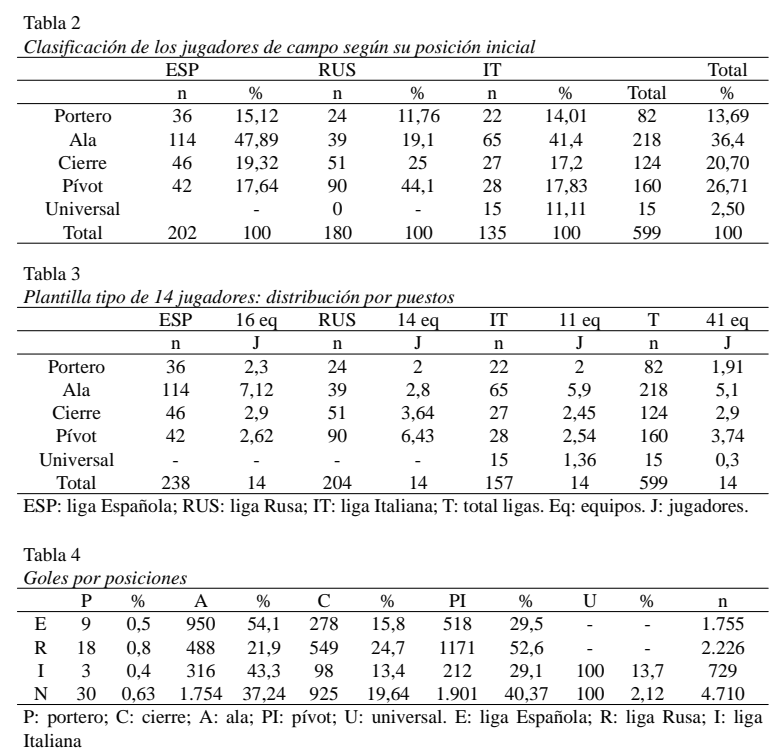

\section{Discusión}

En la discusión de este trabajo se ha de tener en cuenta que no hay muchos estudios de fútbol sala(Moore, Bullough, Goldsmith, \& Edmondson, 2014) y no se ha encontrado ninguno sobre quién mete los goles a excepción de la figura del portero-jugador ya mencionada.

Clasificación de los jugadores según su posición inicial en el campo-confección plantilla

Las posiciones normales iniciales del partido están formadas por cinco jugadores: un portero, un cierre, dos alas y un pívot (figura 1). La posición de ala es la única que es doble por lo que se deberá de tener en cuenta a la hora de discutir los resultados.

Figura 1. Posiciones de jugadores en el campo.

La clasificación de cada jugador participante la hace la liga de ese país. Según los resultados obtenidos (tabla 1) la plantilla tipo de un equipo Europeo formado por 14 jugadores está compuesta por (tabla 2$)$ un 13,69\% $(1,91)$ porteros, $36,4 \%(5,1)$ alas, 20,7\% (2,9) cierres, 26,71\% (3,74) pívots y $2,5 \%(0,3)$ universal. Teniendo en cuenta que la posición de ala es doble (se divide por dos) se puede decir que en términos relativos y absolutos los mayores valores los obtiene la figura del pívot. Este resultado puede considerarse lógico si tenemos en cuenta que este jugador se define como el jugador más próximo a la portería adversaria en el que predominan características ofensivas, como un buen tiro, regate, capacidad de asistir a compañeros, realizar desmarques, buscar finalizar y conseguir goles, objetivo del deporte.

Teniendo en cuenta únicamente la clasificación de los jugadores hechas por las ligas los resultados obtenidos en la liga española e italiana son muy parecidos repartiéndose la figura de jugador universal de la liga italiana entre el cierre y ala en la española (tabla 2). Sin embargo, al comparar la liga española y rusa encontramos grandes diferencias significativas con un 28,8\% más de alas en España que en Rusia y un 26,5\% más de pívots en Rusia que en España. Con respecto a la plantilla tipo estaría formada en España por 2,3 porteros, 7,12 alas, 2,9 cierres y 2,62 pívots mientras que en Rusia estaría formada por 2 porteros, 2,8 alas, 3,64 cierres y 6,43 pívots.

La diferencia entre alas y pívots puede indicar una apuesta distinta en el estilo de juego que se ve ya reflejada en la confección de la plantilla con mayor presencia de unos tipos de jugadores sobre otros.

En la liga rusa, con gran presencia de pívots (44,1\%), se busca un perfil eminentemente ofensivo, quizás buscando la esencia del espectáculo(Cachón, Campoy, Linares, Rodrigo, \& Zagalaz, 2012) que sin duda en el fútbol sala culmina en la finalización y consecución de los goles. Este perfil dominante de jugador ofensivo condiciona el estilo de juego siendo probablemente una liga donde se consigan y encajen muchos goles dando prioridad a un juego ofensivo, ya que el gol es lo más atractivo para el espectador y para el espectáculo (Álvarez, Murillo, \& García, 2018; Cachón, Campoy, Linares, Rodrigo, \& Zagalaz, 2012; Cachón, Valdivia, Lara, Zagalaz, \& Berdejo, 2014). Este estilo de juego estaría en la línea de que el índice de aprovechamiento (goles) es el mejor predictor de rendimiento en el fútbol sala (Lago, Cancela, López, Fernández, \& Veiga, 2006), dando más importancia a los goles que se meten que a los que se encajan. En esta línea, Álvarez, Puente, Manero, \& Manonelles (2004), analizan la relación entre la clasificación final de la temporada 2.002-2.003 de la liga nacional de fútbol sala española con los goles anotados y obtiene que cinco de los equipos más goleadores acaban entre los ocho primeros, mientras que dos de los tres menos realizadores quedaron último y penúltimo en la clasificación. Concluyendo que existe una relación directa entre los goles anotados y la clasificación final.

Por otro lado, la liga española (e italiana) con una mayor presencia de alas y universal, obtiene una distribución más racional, lo que puede indicar un estilo de juego más equilibrado entre ataque y defensa donde quizás no prima tanto los goles que se hagan, sino la relación goles anotados/ encajados. Para poder seguir entendiendo estos resultados es necesario pasar a relacionar esta variable clasificación jugadores con quiénes consiguen los goles.

\section{Goles por posiciones}

Los porteros consiguen el 0,63\% de los goles (30), los alas un 37,24\% (1.754), los cierres un 19,64\% (925), los pívots un 40,37\% (1.901) y los universales un 2,12\% (100) (tabla 4).

Los resultados muestran como las clasificaciones de las plantillas hechas en función de sus características y posi- 
ción inicial en la pista se relacionan directamente con los goles obtenidos, siendo las posiciones con características más ofensivas y más cercanas a la portería contraria las que mayores porcentajes obtienen disminuyendo conforme se alejan. Así, el pívot tiene la mayor responsabilidad obteniendo un $40,37 \%$ de los goles, seguido por los alas con un $37,24 \%$, los cierres con un 19,64\% y los porteros con un 0,63 . Mención especial, por darse solo en la liga italiana, merecen los universales con un 2,12\%.

Al comparar los valores obtenidos en las ligas obtenemos que mientras la liga rusa obtiene el mayor porcentaje de goles en los pívot con un 52,6\%, en la liga española e italiana se da en los alas 54,1\% y 43,3\% respectivamente. Los resultados están relacionados con el porcentaje del tipo de jugador predominante en cada liga, siendo en la rusa los pívots con un 44,1\% y en España e Italia los alas con un 47,89\% y $41,4 \%$ respectivamente. De nuevo, los resultados entre la liga española e italiana son muy similares, repartiéndose los goles del universal de la liga italiana entre el ala y cierre de la española.

En la liga española destacar el 24,6\% más de los goles obtenidos por los alas que por los pívots lo que indica una gran proyección y finalización ofensiva de estos jugadores, repartiendo de esta manera la responsabilidad de finalización entre alas y pívots fundamentalmente. En la liga rusa los pívots obtienen un $30,7 \%$ más de los goles que los alas lo que indica que el pívot es la gran referencia en la finalización ofensiva. Destaca en la liga rusa el cierre que obtiene un porcentaje superior a las alas, lo cual es extraño al ocupar la posición más retrasada de los jugadores de campo. Sería interesante estudiar en qué tipo de jugadas consigue los goles ya que puede ser que sean jugadas que empiezan a balón parado como saques de banda, corners, faltas y dobles penaltis.

Al comparar la liga española y rusa y mismo puesto, la liga española obtiene un 32,2\% más de goles obtenidos por los alas que la rusa y la rusa un 23,1\% más de goles obtenidos por los pívots que la española. Los resultados vuelven a demostrar un estilo de juego diferente.

Todos los resultados corroboran que, pese a la gran movilidad de los jugadores en este deporte, el posicionamiento inicial está relacionado con los goles, a mayor posicionamiento cercano a la portería contraria mayor número de goles. Los resultados obtenidos vuelven a ratificar un estilo de juego diferente entre la liga rusa y la española (e italiana), más ofensivo en la rusa y más equilibrado ataque-defensa en la española e italiana, lo que se traduce en un mayor número y uso de pívots en la liga rusa.

Un reciente estudio(Kacem, Guemri, Naffeti, \& Elloumi, 2016) que analiza el Campeonato del Mundo 2.012 y la Eurocopa de 2.014 confirma que los equipos que más goles meten son los que ganan, estableciendo las probabilidades de ganar en un $75 \%$ si se marcan 4 goles por el $5 \%$ de perder. Se ha de tener en cuenta, que el estilo de juego puede ser muy diferente en un campeonato largo (liga), donde lo más importante es la continuidad de los resultados a lo largo de los meses que uno corto basado en clasificaciones y eliminaciones muchas veces directas. Los resultados nos arrojan nuevas preguntas como ¿tener más pívots en plantilla implicará una mayor media goleadora y aumentar las posibilida- des de ganar los partidos?

Para entender mejor la influencia de cada jugador en la consecución de los goles se necesitan más estudios observacionales que investiguen y relacionen la variable tipo de jugador con sistema y estilos de juego, zona de finalización, etcétera.

\section{Conclusiones}

1. Existe una relación directa entre las posiciones de partida y la consecución de goles. Así, el pívot obtiene un $40,37 \%$ de los goles, seguido por los alas con un $37,24 \%$, los cierres con un $19,64 \%$ y los porteros con un $0,63 \%$. Mención especial, por darse solo en la liga italiana, merecen los universales con un $2,12 \%$.

2. Existen diferencias significativas para todos los jugadores de campo entre la liga rusa y la española. En Rusia el 44,1\% de los jugadores están clasificados como pívots y el 19,01\% como alas mientras en España el 17,64\% están clasificados como pívots y el 47,89\% como alas.

3. Las diferentes confecciones de las plantillas se verán reflejadas en un estilo de juego ofensivo diferente con un gran uso de los pívots en la liga rusa, que obtienen el $52,6 \%$ de los goles y en España de los alas con un 54,1\% de los goles.

4. Los resultados obtenidos muestran diferencias entre las liga española, rusa e italiana lo que influye en el estilo de juego del fútbol sala en esa liga y la efectividad de los jugadores según sus posiciones.

\section{Referencias}

Álvarez, J., Manonelles, P, Giménez, L., \& Nuviala,A. (2009). Incidencia lesional y su repercusión en la planificación del entrenamiento en fútbol sala. Archivos de Medicina del Deporte, 132 (4), 261-272.

Álvarez, J., Murillo, V., \& García, A. (2018). Influencia de la modificación del reglamento en la consecución de los goles en el fútbol sala. Rev.int.med.cienc.act.fís.deporte, 18(70), 213-216.

Álvarez, J., Murillo, V., García,A., \& Parra, A. (2018). Análisis observacional de los goles de dos temporadas de la LNFS. Rev.int.med.cienc.act.fís.deporte, 18, (69), 27-42.

Álvarez, J., Puente, J., Manero, J., \& Manonelles, P. (2004). Análisis de las acciones ofensivas que acaban en gol de la liga profesional de fútbol-sala española. Revista de Entrenamiento Deportivo, 17 (4), 27-32.

Álvarez, J., Ramírez, J., Murillo, V. (2019). El gol como unidad de medida del rendimiento en futsal. Retos, 36, 251-258.

Álvarez, L. (2015). Análisis técnico-táctico de la fase ofensiva de la selección española de fútbol sala (Eurocopa, 2010). Tesis doctoral, Universidad de La Rioja, Logroño.

Argudo, F.M., García, L., \& Ruiz, E. (2016). Factores asociados a la eficacia de gol en waterpolo. Retos, 29, 105-108.

Cachón, J., Campoy, T., Linares, D., Rodrigo, M., \& Zagalaz, M. L. (2012). Análisis cualitativo del cambio de las reglas de juego del fútbol sala (2006) en España. Ágora para la Educación Física y el Deporte, 14 (3), 332-347.

Cachón, J., Valdivia, P., Lara, A., Zagalaz, M. L., \& Berdejo, D. (2014). Questionnaire: Loss of Entertainment in Spanish 
Futsal (PEFSE)-Results Analysis. American Journal of Sports Science and Medicine, 2 (3), 83-87.

Campos, F., Pellegrinotti, I., Pasquarelli, B., Rabelo, F., Santa Cruz, R., \& Gómez, MA. (2015). Effects of game-location and quality of opposition in futsal league. International Journal of Performance Analysis in Sport, 15, 598-607.

Da Cuna Voser, R., Goncalves, C., \& Eloi, P. (2016). The origin of Futsal 2014 League goals. Revista Brasileira de Futsal e Futebol, 8(29), 155-160.

Grela, I. (2013). Analysis of the goals in season 2012-2013 in the first división (lnfs) depending on the time and the match and result. Revista fútbol táctico, 78, 177-183.

Jiménez-Olmedo, J.M., Espina-Agullo, J.J., \& Manchado, C. (2017). Análisis histórico de la efectividad de los lanzamientos a portería de balonmano masculino. Retos, 32, 228-232.

Kacem, N., Guemri, A., Naffeti, C., \& Elloumi, A. (2016). Mechanism of Social Reproduction of the Culture Futsal: Modelling of the Universals of Futsal and Sense of the Rules of the Game: Analysis of Shooting at the European Cup Matches. Advances in Physical Education, 6, 5966.

Lago, C., Cancela, J. M., López, M. D. P., Fernández, F., \& Veiga, J. (2006). Evaluation of offensive actions in football performance against indicators of success in intensive diachronic retrospective designs, Apunts: Educación física y deportes. 12 (72), 96-103.
Lapresa, D., Álvarez, L., Arana, J., Garzón, B., \& Caballero, V. (2013). Observational analysis of the offensive sequences that ended in ashot by the winning team of the 2010 UEFA Futsal Championship. Journal of Sports Sciences, 31(15), 1731-1739.

Martínez, F.D., \& González, H. (2019). Efecto de marcar primero y la localización del partido en las principales ligas del fútbol europeo. Retos, 35, 242-245.

Moore, R., Bullough, S., Goldsmith, S., \& Edmondson, L. (2014). A Systematic review of Futsal Literature. American Journal of Sport Science and Medicine, 2 (3), 108-116.

Pascual, V., Álvarez, A., Carbonel, J.A., \& Pérez, J.A. (2019). Análisis de patrón gol en competición de fútbol sala: $1^{\text {a }}$ División y 2a División B. Retos, 35, 364-368.

Sampedro, J., \& Prieto, J. (2011). La territorialidad como factor asociado a la ventaja de jugar en casa. Un estudio comparativo por regiones en la liga de fútbol y en la liga de fútbol sala de España. European Journal of Human Movement. 26, 93-104.

Schneider, I., Da Cunha Voser, R., \& Eloi, P. (2015). Analysis of goals scored and suffered by the under- 17 futsal team from Nova Itaberaba-SC during the Santa Cataria championship 2013-2014. Revista Brasileira de Futsal e Futebol, 7(5), 327-331.

Spanish Federation of Football (RFEF). (2009). Laws of the Game Futsal. Madrid: RFEF.

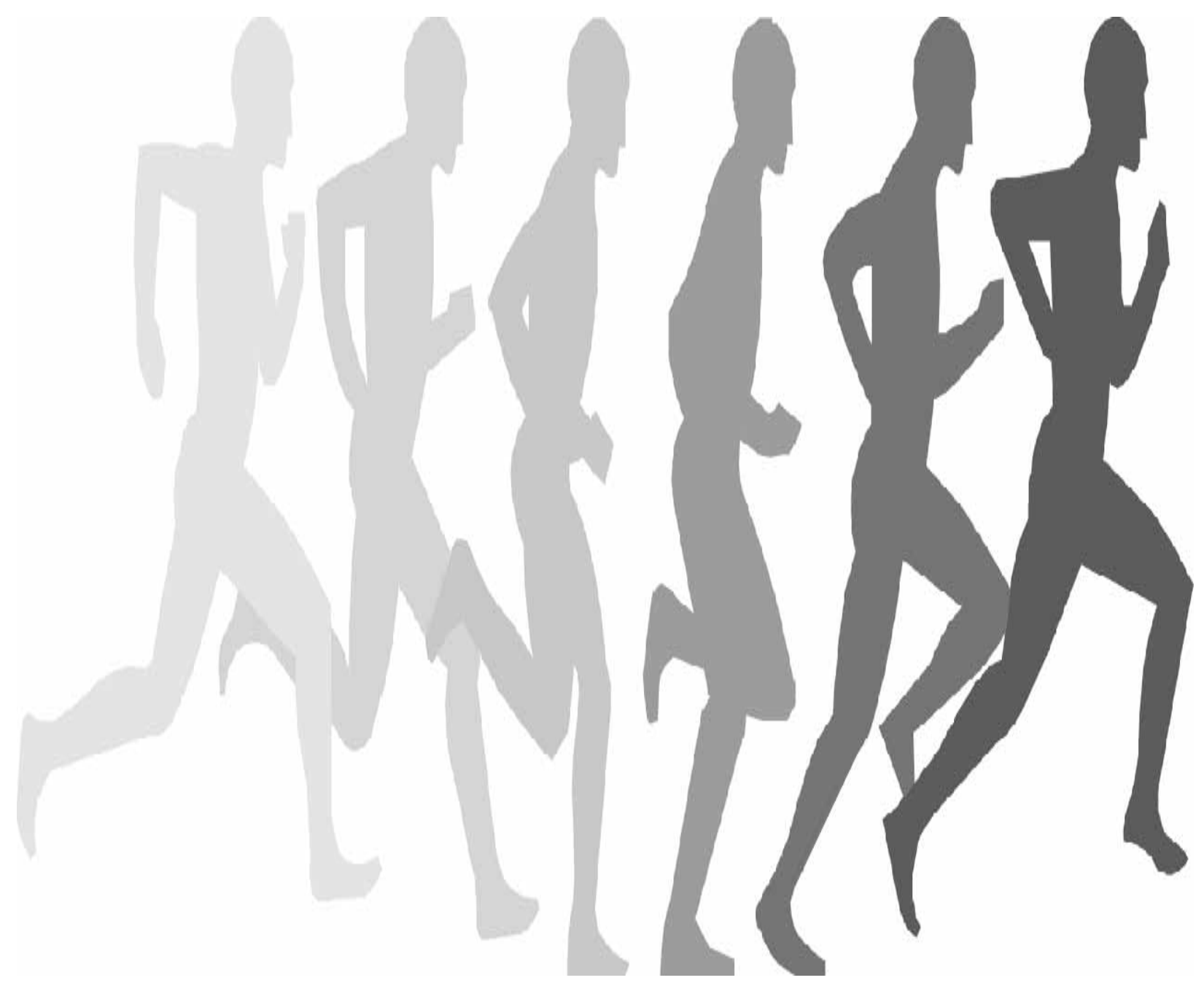

